\title{
Necessary and sufficient conditions for the two parameter generalized Wilker-type inequalities
}

Hui Sun ${ }^{1}$, Zhen-Hang Yang ${ }^{2}$ and Yu-Ming Chu ${ }^{1 *}$

${ }^{\text {"Correspondence: }}$
chuyuming2005@126.com
${ }^{1}$ School of Mathematics and
Computation Sciences, Hunan City
University, Yiyang, 413000, China
Full list of author information is
available at the end of the article

\section{Abstract}

In the article, we provide the necessary and sufficient conditions for the parameters $\alpha$ and $\beta$ such that the generalized Wilker-type inequality

$$
\frac{2 \beta}{\alpha+2 \beta}\left(\frac{\sin x}{x}\right)^{\alpha}+\frac{\alpha}{\alpha+2 \beta}\left(\frac{\tan x}{x}\right)^{\beta}-1>(<) 0
$$

holds for all $x \in(0, \pi / 2)$.

MSC: 26D05; 33B10

Keywords: Wilker-type inequality; sine function; tangent function; necessary and sufficient condition

\section{Introduction}

The Wilker inequality $[1,2]$ for sine and tangent functions states that the inequality

$$
\left(\frac{\sin x}{x}\right)^{2}+\frac{\tan x}{x}-2>0
$$

holds for all $x \in(0, \pi / 2)$. The generalizations and improvements for the Wilker inequality (1.1) have been the subject of intensive research in the recent years. Wu and Srivastava [3] proved that the inequality

$$
\frac{\lambda}{\lambda+\mu}\left(\frac{\sin x}{x}\right)^{p}+\frac{\mu}{\lambda+\mu}\left(\frac{\tan x}{x}\right)^{q}>1
$$

holds for all $x \in(0, \pi / 2)$ if $\lambda>0, \mu>0, q>0$ or $q \leq \min \{-1,-\lambda / \mu\}$, and $p \leq 2 q \mu / \lambda$. Baricz and Sándor [4] generalized inequality (1.2) to the Bessel functions.

In [5], Zhu proved that the inequalities

$$
\left(\frac{\sin x}{x}\right)^{2 p}+\left(\frac{\tan x}{x}\right)^{p}>\left(\frac{x}{\sin x}\right)^{2 p}+\left(\frac{x}{\tan x}\right)^{p}>2
$$

hold for $x \in(0, \pi / 2)$ and $p \geq 1$. Matejíčka [6] presented the best possible parameter $p$ such that the second inequality of (1.3) holds for $x \in(0, \pi / 2)$.

(c) The Author(s) 2016. This article is distributed under the terms of the Creative Commons Attribution 4.0 International License (http://creativecommons.org/licenses/by/4.0/), which permits unrestricted use, distribution, and reproduction in any medium, provided you give appropriate credit to the original author(s) and the source, provide a link to the Creative Commons license, and indicate if changes were made. 
Zhu [7] proved that the inequalities

$$
(1-\lambda)\left(\frac{x}{\sin x}\right)^{p}+\lambda\left(\frac{x}{\tan x}\right)^{p}<1<(1-\eta)\left(\frac{x}{\sin x}\right)^{p}+\eta\left(\frac{x}{\tan x}\right)^{p}
$$

are valid for all $x \in(0, \pi / 2)$ if $(p, \lambda, \eta) \in\left\{(p, \lambda, \eta) \mid p \geq 1, \lambda \geq 1-(2 / \pi)^{p}, \eta \leq 1 / 3\right\} \cup$ $\left\{(p, \lambda, \eta) \mid 0 \leq p \leq 4 / 5, \lambda \geq 1 / 3, \eta \leq 1-(2 / \pi)^{p}\right\}$.

In [8], Yang and Chu provided the necessary and sufficient condition for the parameter $\mu$ such that the generalized Wilker-type inequality

$$
\frac{2}{\lambda+2}\left(\frac{\sin x}{x}\right)^{\lambda \mu}+\frac{\lambda}{\lambda+2}\left(\frac{\tan x}{x}\right)^{\mu}-1>(<) 0
$$

holds for any fixed $\lambda \geq 1$ and all $x \in(0, \pi / 2)$.

Very recently, Chu et al. [9] proved that the two parameter generalized Wilker-type inequality

$$
\frac{2 \beta}{\alpha+2 \beta}\left(\frac{\sin x}{x}\right)^{\alpha}+\frac{\alpha}{\alpha+2 \beta}\left(\frac{\tan x}{x}\right)^{\beta}-1>0
$$

holds for all $x \in(0, \pi / 2)$ if $(\alpha, \beta) \in E_{0}$, and inequality (1.4) is reversed if $(\alpha, \beta) \in E_{1}$, where

$$
\begin{aligned}
E_{0}= & \{(\alpha, \beta) \mid \alpha>0, \beta>0\} \cup\{(\alpha, \beta) \mid 0<\alpha<-2 \beta, \beta \geq-1\} \\
& \cup\left\{(\alpha, \beta) \mid \beta>0,-\frac{12}{5} \leq \alpha+2 \beta<0\right\} \\
& \cup\left\{(\alpha, \beta) \mid \alpha \leq \frac{\pi^{2}}{4}-3, \beta \leq-1\right\} \\
& \cup\left\{(\alpha, \beta) \mid \frac{\pi^{2}}{4}-3<\alpha<0, \beta \leq-\frac{37}{35}, \alpha+2 \beta+\frac{12}{5} \leq 0\right\}, \\
E_{1}= & \{(\alpha, \beta) \mid \alpha<0, \alpha+2 \beta>0\} \cup\{(\alpha, \beta) \mid-1 \leq \beta<0, \alpha+2 \beta>0\} \\
& \cup\left\{(\alpha, \beta) \mid-1 \leq \beta<0,-2 \beta-\frac{12}{5} \leq \alpha<0\right\} \cup\left\{(\alpha, \beta) \mid 0<\alpha \leq-2 \beta-\frac{12}{5}\right\} .
\end{aligned}
$$

The main purpose of this paper is to provide the necessary and sufficient conditions for the parameters $\alpha$ and $\beta$ such that the generalized Wilker-type inequality (1.4) and its reversed inequality hold for all $x \in(0, \pi / 2)$.

\section{Lemmas}

Lemma 2.1 (See [10], Lemma 2.3) Let $-\infty<\alpha<\beta<\infty, f_{1}, f_{2}:[\alpha, \beta] \rightarrow \mathbb{R}$ be continuous on $[\alpha, \beta]$ and differentiable on $(\alpha, \beta)$, and $f_{2}^{\prime}(x) \neq 0$ on $(\alpha, \beta)$. Then the inequality

$$
\frac{f_{1}(x)-f_{1}(\alpha)}{f_{2}(x)-f_{2}(\alpha)}>(<) \frac{f_{1}^{\prime}\left(\alpha^{+}\right)}{f_{2}^{\prime}\left(\alpha^{+}\right)}
$$

holds for all $x \in(\alpha, \beta)$ if there exists $\eta \in(\alpha, \beta)$ such that $f_{1}^{\prime}(x) / f_{2}^{\prime}(x)$ is strictly increasing (decreasing) on $(\alpha, \eta)$ and strictly decreasing (increasing) on $(\eta, \beta)$, and

$$
\frac{f_{1}(\beta)-f_{1}(\alpha)}{f_{2}(\beta)-g_{2}(\alpha)} \geq(\leq) \frac{f_{1}^{\prime}\left(\alpha^{+}\right)}{f_{2}^{\prime}\left(\alpha^{+}\right)} \neq \infty
$$


Lemma 2.2 (See [9], Lemma 2.9) Let $\beta \in \mathbb{R}, x \in(0, \pi / 2)$, and $\mathrm{F}(x), \mathrm{G}(x), \mathrm{H}(x)$ and $g(x)$ be defined by

$$
\begin{aligned}
& \mathrm{F}(x)=\cos x(\sin x-x \cos x)^{2}(x-\sin x \cos x), \\
& \mathrm{G}(x)=(x-\sin x \cos x)^{2}(\sin x-x \cos x), \\
& \mathrm{H}(x)=x^{3}\left(\frac{\sin ^{2} x}{x^{2}}+\frac{\tan x}{x}-2\right) \sin ^{2} x \cos x
\end{aligned}
$$

and

$$
g(x)=\frac{\beta \mathrm{G}(x)+\mathrm{H}(x)}{\mathrm{F}(x)},
$$

respectively. Then the following statements are true:

(1) The function $g(x)$ is strictly increasing from $(0, \pi / 2)$ onto $\left(2 \beta+12 / 5,3-\pi^{2} / 4\right)$ if $\beta=-1$.

(2) The function $g(x)$ is strictly increasing from $(0, \pi / 2)$ onto $(2 \beta+12 / 5, \infty)$ if $\beta>-1$.

(3) The function $g(x)$ is strictly decreasing from $(0, \pi / 2)$ onto $(-\infty, 2 \beta+12 / 5)$ if $\beta \leq-37 / 35$.

Let $\alpha, \beta \in \mathbb{R}, x \in(0, \pi / 2)$ and the functions $\mathrm{I}_{\alpha}(x), \mathrm{J}_{\beta}(x)$ and $\mathrm{Q}_{\alpha, \beta}(x)$ be defined by

$$
\begin{array}{lll}
\mathrm{I}_{\alpha}(x)=\frac{1-\left(\frac{\sin x}{x}\right)^{\alpha}}{\alpha} \quad(\alpha \neq 0), & \mathrm{I}_{0}(x)=\log x-\log (\sin x), \\
\mathrm{J}_{\beta}(x)=\frac{\left(\frac{\tan x}{x}\right)^{\beta}-1}{\beta} \quad(\beta \neq 0), & \mathrm{J}_{0}(x)=\log (\tan x)-\log x,
\end{array}
$$

and

$$
\mathrm{Q}_{\alpha, \beta}(x)=\frac{\mathrm{I}_{\alpha}(x)}{\mathrm{J}_{\beta}(x)}
$$

respectively.

Then it is not difficult to verify that

$$
\begin{aligned}
& \mathrm{I}_{\alpha}\left(0^{+}\right)=\mathrm{J}_{\beta}\left(0^{+}\right)=0, \\
& \mathrm{Q}_{\alpha, \beta}(x)=\frac{\mathrm{I}_{\alpha}(x)}{\mathrm{J}_{\beta}(x)}=\frac{\mathrm{I}_{\alpha}(x)-\mathrm{I}_{\alpha}\left(0^{+}\right)}{\mathrm{J}_{\beta}(x)-\mathrm{J}_{\beta}\left(0^{+}\right)}, \\
& \mathrm{Q}_{\alpha, \beta}\left(0^{+}\right)=\frac{1}{2}, \\
& \mathrm{Q}_{\alpha, \beta}\left(\frac{\pi}{2}\right)=\frac{\beta}{\alpha}\left[\left(\frac{2}{\pi}\right)^{\alpha}-1\right] \quad(\alpha \neq 0, \beta<0), \\
& \mathrm{Q}_{0, \beta}\left(\frac{\pi^{-}}{2}\right)=\lim _{\alpha \rightarrow 0} Q_{\alpha, \beta}\left(\frac{\pi^{-}}{2}\right)=\beta \log \frac{2}{\pi} \quad(\beta<0) .
\end{aligned}
$$

Lemma 2.3 (See [9], Lemma 2.10) Let $x \in(0, \pi / 2)$ and $\mathrm{Q}_{\alpha, \beta}(x)$ be defined by (2.7). Then the following statements are true: 
(1) If $\alpha+2 \beta+12 / 5 \geq 0$ and $\beta \geq-1$, then $\mathrm{Q}_{\alpha, \beta}(x)$ is strictly decreasing on $(0, \pi / 2)$.

(2) If $\alpha \leq \pi^{2} / 4-3$ and $-37 / 35<\beta \leq-1$, then $\mathrm{Q}_{\alpha, \beta}(x)$ is strictly increasing on $(0, \pi / 2)$.

(3) If $\alpha+2 \beta+12 / 5 \leq 0$ and $\beta \leq-37 / 35$, then $\mathrm{Q}_{\alpha, \beta}(x)$ is strictly increasing on $(0, \pi / 2)$.

Lemma 2.4 Let $x \in(0, \pi / 2), \mathrm{Q}_{\alpha, \beta}(x)$ be defined by (2.7) and the function $x \rightarrow \mathrm{D}(\alpha, \beta ; x)$ be defined by

$$
\mathrm{D}(\alpha, \beta ; x)=\mathrm{Q}_{\alpha, \beta}(x)-\frac{1}{2} .
$$

Then the following statements are true:

(1) If $\alpha \in \mathbb{R}$ is fixed and $\beta<0$, then there exists a unique solution $\beta=\beta(\alpha)$ given by

$$
\beta(\alpha)=\frac{\alpha}{2\left[\left(\frac{2}{\pi}\right)^{\alpha}-1\right]} \quad(\alpha \neq 0), \quad \beta(0)=\frac{1}{2 \log \frac{2}{\pi}}
$$

satisfies the equation $\mathrm{D}\left(\alpha, \beta ; \frac{\pi}{2}^{-}\right)=0$ such that $\mathrm{D}\left(\alpha, \beta ; \frac{\pi}{2}^{-}\right)>0$ for $\beta<\beta(\alpha)$ and $\mathrm{D}(\alpha, \beta$; $\left.\frac{\pi}{2}^{-}\right)<0$ for $\beta>\beta(\alpha)$.

(2) If $\beta<0$ is fixed, then there exists a unique solution $\alpha=\alpha(\beta)$ satisfies the equation $\mathrm{D}\left(\alpha, \beta ; \frac{\pi}{2}^{-}\right)=0$ such that $\mathrm{D}\left(\alpha, \beta ; \frac{\pi}{2}^{-}\right)>0$ for $\alpha<\alpha(\beta)$ and $\mathrm{D}\left(\alpha, \beta ; \frac{\pi}{2}^{-}\right)<0$ for $\alpha>\alpha(\beta)$. In particular, one has

$$
\alpha_{0}=\alpha(-1)=-0.44367302 \cdots, \quad \alpha_{0}^{*}=\alpha\left(-\frac{37}{35}\right)=-0.20340978 \cdots
$$

(3) The two functions $\alpha \rightarrow \beta(\alpha)$ and $\beta \rightarrow \alpha(\beta)$ are strictly decreasing.

Proof Part (1) follows easily from (2.9)-(2.11) and the fact that $\left[(2 / \pi)^{\alpha}-1\right] / \alpha<0$.

(2) It follows from (2.9) and (2.11) that

$$
\lim _{\alpha \rightarrow-\infty} \mathrm{D}\left(\alpha, \beta ; \frac{\pi^{-}}{2}\right)=\infty, \quad \lim _{\alpha \rightarrow \infty} \mathrm{D}\left(\alpha, \beta ; \frac{\pi^{-}}{2}\right)=-\frac{1}{2} .
$$

Note that

$$
\frac{d}{d \alpha}\left[\frac{\left(\frac{2}{\pi}\right)^{\alpha}-1}{\alpha}\right]=\frac{\left(\frac{2}{\pi}\right)^{\alpha}}{\alpha^{2}}\left[\log \left(\frac{2}{\pi}\right)^{\alpha}+\left(\frac{2}{\pi}\right)^{-\alpha}-1\right]>0
$$

for $\alpha \neq 0$.

From (2.9), (2.11), and (2.15) we clearly see that the function $\alpha \rightarrow \mathrm{D}\left(\alpha, \beta ; \frac{\pi}{2}^{-}\right)$is strictly decreasing. Therefore, there exists a unique solution $\alpha=\alpha(\beta)$ that satisfies the equation $\mathrm{D}\left(\alpha, \beta ; \frac{\pi}{2}^{-}\right)=0$ such that $\mathrm{D}\left(\alpha, \beta ; \frac{\pi}{2}^{-}\right)>0$ for $\alpha<\alpha(\beta)$ and $\mathrm{D}\left(\alpha, \beta ; \frac{\pi}{2}^{-}\right)<0$ for $\alpha>\alpha(\beta)$ follows from (2.14) and the monotonicity of the function $\alpha \rightarrow \mathrm{D}\left(\alpha, \beta ; \frac{\pi}{2}^{-}\right)$. Numerical computations show that

$$
\alpha(-1)=-0.44367302 \cdots, \quad \alpha\left(-\frac{37}{35}\right)=-0.20340978 \cdots
$$

(3) The function $\alpha \rightarrow \beta(\alpha)$ is strictly decreasing follows easily from (2.12) and (2.15). The function $\beta \rightarrow \alpha(\beta)$ is strictly decreasing due to it is the inverse function of $\alpha \rightarrow \beta(\alpha)$. 
Lemma 2.5 Let $\beta(\alpha)$ be defined by (2.12). Then

$$
\alpha_{1}=-0.36131140 \cdots
$$

is the unique solution of the equation $\beta(\alpha)=-\alpha / 2-6 / 5$ such that $\beta(\alpha)<-\alpha / 2-6 / 5$ for $\alpha<\alpha_{1}$ and $\beta(\alpha)>-\alpha / 2-6 / 5$ for $\alpha>\alpha_{1}$.

Proof Let $P(\alpha)=\beta(\alpha)+\alpha / 2+6 / 5$. Then from (2.12) we clearly see that

$$
\begin{aligned}
& P(\alpha)=\frac{\left(\frac{2}{\pi}\right)^{\alpha}}{2} \frac{\alpha}{\left(\frac{2}{\pi}\right)^{\alpha}-1}+\frac{6}{5}, \\
& \lim _{\alpha \rightarrow-\infty} P(\alpha)=-\infty, \quad \lim _{\alpha \rightarrow \infty} P(\alpha)=\frac{6}{5}, \\
& \frac{d P(\alpha)}{d \alpha}=-\frac{\left(\frac{2}{\pi}\right)^{\alpha}}{2} \frac{\log \left(\frac{2}{\pi}\right)^{\alpha}-\left(\frac{2}{\pi}\right)^{\alpha}+1}{\left[\left(\frac{2}{\pi}\right)^{\alpha}-1\right]^{2}}>0
\end{aligned}
$$

for $\alpha \neq 0$, where the last of (2.18) due to $\log x-x+1<0$ for all $x>0$ with $x \neq 1$.

Inequality (2.18) implies that the function $\alpha \rightarrow P(\alpha)$ is strictly increasing on $(0, \infty)$. Therefore, there exists a unique $\alpha=\alpha_{1}$ that satisfies the equation $\beta(\alpha)=-\alpha / 2-6 / 5$ such that $\beta(\alpha)<-\alpha / 2-6 / 5$ for $\alpha<\alpha_{1}$ and $\beta(\alpha)>-\alpha / 2-6 / 5$ for $\alpha>\alpha_{1}$ follows from (2.17) and the monotonicity of the function $\alpha \rightarrow P(\alpha)$. Numerical computations show that $\alpha_{1}=-0.36131140 \cdots$.

Lemma 2.6 Let $\mathrm{Q}_{\alpha, \beta}(x), \beta(\alpha), \alpha_{0}$ and $\alpha_{0}^{*}$ be defined by (2.7), (2.12), and (2.13), respectively. Then the following statements are true:

(1) If $\alpha \geq-2 / 7=-0.28571428 \cdots$, then the inequality $\mathrm{Q}_{\alpha, \beta}(x)>1 / 2$ holds for all $x \in(0, \pi / 2)$ if and only if $\beta \leq-\alpha / 2-6 / 5$.

(2) If $\alpha \geq \alpha_{0}^{*}$, then the inequality $\mathrm{Q}_{\alpha, \beta}(x)<1 / 2$ holds for all $x \in(0, \pi / 2)$ if and only if $\beta \geq \beta(\alpha)$.

(3) If $\alpha \leq-2 / 5$, then the inequality $\mathrm{Q}_{\alpha, \beta}(x)<1 / 2$ holds for all $x \in(0, \pi / 2)$ if and only if $\beta \geq-\alpha / 2-6 / 5$.

(4) If $\alpha \leq \alpha_{0}$, then the inequality $\mathrm{Q}_{\alpha, \beta}(x)>1 / 2$ holds for all $x \in(0, \pi / 2)$ if and only if $\beta \leq \beta(\alpha)$.

Proof (1) If $\alpha \geq-2 / 7$ and $\mathrm{Q}_{\alpha, \beta}(x)>1 / 2$ for all $x \in(0, \pi / 2)$, then from (2.5)-(2.7) one has

$$
\lim _{x \rightarrow 0^{+}} x^{-2}\left[\mathrm{Q}_{\alpha, \beta}(x)-\frac{1}{2}\right]=\lim _{x \rightarrow 0^{+}} x^{-2}\left[-\frac{5 \alpha+10 \beta+12}{120} x^{2}+o\left(x^{2}\right)\right]=-\frac{5 \alpha+10 \beta+12}{120} \geq 0
$$

which implies that $\beta \leq-\alpha / 2-6 / 5$.

If $\alpha \geq-2 / 7$ and $\beta \leq-\alpha / 2-6 / 5$, then we clearly see

$$
\alpha+2 \beta+\frac{12}{5} \leq 0, \quad \beta \leq-\frac{37}{35} .
$$

Therefore, $\mathrm{Q}_{\alpha, \beta}(x)>1 / 2$ for all $x \in(0, \pi / 2)$ follows from Lemma 2.3(3) and (2.8) together with (2.19). 
(2) If $\alpha \geq \alpha_{0}^{*}$ and $\mathrm{Q}_{\alpha, \beta}(x)<1 / 2$ for all $x \in(0, \pi / 2)$, then from (2.11) and Lemma 2.4(1) we clearly see that $\mathrm{D}\left(\alpha, \beta ; \frac{\pi}{2}^{-}\right) \leq 0$ and $\beta \geq \beta(\alpha)$.

Next, we prove that $\mathrm{Q}_{\alpha, \beta}(x)<1 / 2$ for all $x \in(0, \pi / 2)$ if $\alpha \geq \alpha_{0}^{*}$ and $\beta \geq \beta(\alpha)$. It follows from (2.6) and (2.7) together with the fact that

$$
\frac{\partial J_{\beta}(x)}{\partial \beta}=\frac{\left(\frac{\tan x}{x}\right)^{\beta}}{\beta^{2}}\left[\log \left(\frac{\tan x}{x}\right)^{\beta}+\left(\frac{x}{\tan x}\right)^{\beta}-1\right]>0
$$

for $x \in(0, \pi / 2)$ and $\beta \neq 0$ that the function $\beta \rightarrow \mathrm{Q}_{\alpha, \beta}(x)$ is strictly decreasing. Therefore, it suffices to prove that $\mathrm{Q}_{\alpha, \beta}(x)<1 / 2$ for all $x \in(0, \pi / 2)$ if $\alpha \geq \alpha_{0}^{*}$ and $\beta=\beta(\alpha)$.

From (2.13) and Lemma 2.4(3) we get

$$
\beta=\beta(\alpha) \leq \beta\left(\alpha_{0}^{*}\right)=-\frac{37}{35} .
$$

Let $\alpha \beta \neq 0, \mathrm{~F}(x), \mathrm{G}(x), \mathrm{H}(x), g(x), \mathrm{I}_{\alpha}(x)$ and $\mathrm{J}_{\beta}(x)$ be defined by (2.1)-(2.6), respectively. Then simple computations lead to

$$
\begin{aligned}
& {\left[\frac{\mathrm{I}_{\alpha}^{\prime}(x)}{\mathrm{J}_{\beta}^{\prime}(x)}\right]^{\prime}=-\frac{\cos ^{\beta} x \sin ^{\alpha-\beta-1} x}{(x-\sin x \cos x)^{2}}[g(x)+\alpha] \mathrm{F}(x) x^{\beta-\alpha-1},} \\
& \mathrm{~J}_{\beta}^{\prime}(x)=\frac{2 x-\sin (2 x)}{2 x^{2} \cos ^{2} x}\left(\frac{\tan x}{x}\right)^{\beta-1}>0
\end{aligned}
$$

for $x \in(0, \pi / 2)$.

Let $\alpha_{1}=-0.36131140 \cdots$ be defined by (2.16). Then it follows from Lemma 2.2(3), Lemma 2.5, and (2.20) together with $\alpha \geq \alpha_{0}^{*}=-0.20340978 \cdots>\alpha_{1}$ that the function $x \rightarrow g(x)+\alpha$ is strictly decreasing on $(0, \pi / 2)$ and

$$
\lim _{x \rightarrow 0^{+}}[g(x)+\alpha]=\alpha+2 \beta+\frac{12}{5}>0, \quad \lim _{x \rightarrow \frac{\pi}{2}^{-}}[g(x)+\alpha]=-\infty .
$$

From (2.21) and (2.23) together with the monotonicity of the function $x \rightarrow g(x)+\alpha$ on the interval $(0, \pi / 2)$ we clearly see that there exists $x_{0} \in(0, \pi / 2)$ such that the function $x \rightarrow \mathrm{I}_{\alpha}^{\prime}(x) / J_{\beta}^{\prime}(x)$ is strictly decreasing on $\left(0, x_{0}\right)$ and strictly increasing on $\left(x_{0}, \pi / 2\right)$.

Note that

$$
\frac{\mathrm{I}_{\alpha}\left(\frac{\pi}{2}^{-}\right)-\mathrm{I}_{\alpha}\left(0^{+}\right)}{\mathrm{J}_{\beta(\alpha)}\left(\frac{\pi}{2}^{-}\right)-\mathrm{J}_{\beta(\alpha)}\left(0^{+}\right)}=\mathrm{Q}_{\alpha, \beta(\alpha)}\left(\frac{\pi}{2}^{-}\right)=\mathrm{D}\left(\alpha, \beta(\alpha) ; \frac{\pi}{2}^{-}\right)+\frac{1}{2}=\frac{1}{2} .
$$

Therefore, $\mathrm{Q}_{\alpha, \beta}(x)<1 / 2$ for all $x \in(0, \pi / 2)$ follows from Lemma 2.1, (2.7), (2.22), (2.24), and the piecewise monotonicity of the function $x \rightarrow \mathrm{I}_{\alpha}^{\prime}(x) / \mathrm{J}_{\beta}^{\prime}(x)$ on the interval $(0, \pi / 2)$.

(3) If $\alpha \leq-2 / 5$ and $\mathrm{Q}_{\alpha, \beta}(x)<1 / 2$ for all $x \in(0, \pi / 2)$, then from (2.5)-(2.7) we have

$$
\lim _{x \rightarrow 0^{+}} x^{-2}\left[\mathrm{Q}_{\alpha, \beta}(x)-\frac{1}{2}\right]=\lim _{x \rightarrow 0^{+}} x^{-2}\left[-\frac{5 \alpha+10 \beta+12}{120} x^{2}+o\left(x^{2}\right)\right]=-\frac{5 \alpha+10 \beta+12}{120} \leq 0,
$$

which implies that $\beta \geq-\alpha / 2-6 / 5$.

If $\alpha \leq-2 / 5$ and $\beta \geq-\alpha / 2-6 / 5$, then we clearly see that

$$
\alpha+2 \beta+\frac{12}{5} \geq 0, \quad \beta \geq-1
$$


Therefore, $\mathrm{Q}_{\alpha, \beta}(x)<1 / 2$ for $x \in(0, \pi / 2)$ follows easily from Lemma 2.3(1), (2.8), and (2.25).

(4) If $\alpha \leq \alpha_{0}$ and $\mathrm{Q}_{\alpha, \beta}(x)>1 / 2$ for all $x \in(0, \pi / 2)$, then (2.11) and Lemma 2.4(1) lead to the conclusion that $\mathrm{D}\left(\alpha, \beta ; \frac{\pi}{2}^{-}\right) \geq 0$ and $\beta \leq \beta(\alpha)$.

Next, we prove that $\mathrm{Q}_{\alpha, \beta}(x)>1 / 2$ for all $x \in(0, \pi / 2)$ if $\alpha \leq \alpha_{0}$ and $\beta \leq \beta(\alpha)$. Since the function $\beta \rightarrow \mathrm{Q}_{\alpha, \beta}(x)$ is strictly decreasing which was proved in part (2), we only need to prove that $\mathrm{Q}_{\alpha, \beta}(x)>1 / 2$ for all $x \in(0, \pi / 2)$ if $\alpha \leq \alpha_{0}$ and $\beta=\beta(\alpha)$. It follows from Lemma 2.2(1) and (2), Lemma 2.4(3), Lemma 2.5, and $\alpha \leq \alpha_{0}<\alpha_{1}$ that $\beta \geq \beta\left(\alpha_{0}\right)=-1$ and the function $g(x)+\alpha$ is strictly increasing on $(0, \pi / 2)$ such that

$$
\begin{aligned}
\lim _{x \rightarrow 0^{+}}[g(x)+\alpha] & =\alpha+2 \beta+\frac{12}{5}<0, \\
\lim _{x \rightarrow \frac{\pi}{2}^{-}}[g(x)+\alpha] & = \begin{cases}\alpha+\infty, & \beta(\alpha)>-1, \\
\alpha+3-\frac{\pi^{2}}{4}, & \beta(\alpha)=-1,\end{cases} \\
& = \begin{cases}\infty, & \beta>-1, \\
\alpha_{0}+3-\frac{\pi^{2}}{4}>0, & \beta=-1 .\end{cases}
\end{aligned}
$$

From (2.21), (2.26), and (2.27) we clearly see that there exists $x^{*} \in(0, \pi / 2)$ such that the function $x \rightarrow \mathrm{I}_{\alpha}^{\prime}(x) / \mathrm{J}_{\beta}^{\prime}(x)$ is strictly increasing on $\left(0, x^{*}\right)$ and strictly decreasing on $\left(x^{*}, \pi / 2\right)$. Therefore, $\mathrm{Q}_{\alpha, \beta}(x)>1 / 2$ for all $x \in(0, \pi / 2)$ follows from Lemma 2.1, (2.7), (2.22), (2.24), and the piecewise monotonicity of the function $x \rightarrow \mathrm{I}_{\alpha}^{\prime}(x) / \mathrm{J}_{\beta}^{\prime}(x)$ on the interval $(0, \pi / 2)$.

Lemma 2.7 Let $\mathrm{Q}_{\alpha, \beta}(x), \alpha_{0}, \alpha_{0}^{*}$ and $\alpha(\beta)$ be defined by (2.7) and Lemma 2.4, respectively. Then the following statements are true:

(1) If $\beta \geq-1$, then the inequality $\mathrm{Q}_{\alpha, \beta}(x)<1 / 2$ holds for all $x \in(0, \pi / 2)$ if and only if $\alpha \geq-2 \beta-12 / 5$.

(2) If $-1 \leq \beta<0$, then the inequality $\mathrm{Q}_{\alpha, \beta}(x)>1 / 2$ holds for all $x \in(0, \pi / 2)$ if and only if $\alpha \leq \alpha(\beta)$.

(3) If $\beta \leq-37 / 35$, then the inequality $\mathrm{Q}_{\alpha, \beta}(x)>1 / 2$ holds for all $x \in(0, \pi / 2)$ if and only if $\alpha \leq-2 \beta-12 / 5$.

(4) If $\beta \leq-37 / 35$, then the inequality $\mathrm{Q}_{\alpha, \beta}(x)<1 / 2$ holds for all $x \in(0, \pi / 2)$ if and only if $\alpha \geq \alpha(\beta)$.

Proof (1) If $\beta \geq-1$ and $\mathrm{Q}_{\alpha, \beta}(x)<1 / 2$ for all $x \in(0, \pi / 2)$, then from (2.5)-(2.7) we get

$$
\lim _{x \rightarrow 0^{+}} x^{-2}\left[\mathrm{Q}_{\alpha, \beta}(x)-\frac{1}{2}\right]=\lim _{x \rightarrow 0^{+}} x^{-2}\left[-\frac{5 \alpha+10 \beta+12}{120} x^{2}+o\left(x^{2}\right)\right]=-\frac{5 \alpha+10 \beta+12}{120} \leq 0,
$$

which implies that $\alpha \geq-2 \beta-12 / 5$.

If $\beta \geq-1$ and $\alpha \geq-2 \beta-12 / 5$, then $\mathrm{Q}_{\alpha, \beta}(x)<1 / 2$ for all $x \in(0, \pi / 2)$ follows from (2.8) and Lemma 2.3(1).

(2) If $-1 \leq \beta<0$ and $\mathrm{Q}_{\alpha, \beta}(x)>1 / 2$ for all $x \in(0, \pi / 2)$, then (2.11) and Lemma 2.4(2) lead to the conclusion that $\mathrm{D}\left(\alpha, \beta ; \frac{\pi}{2}^{-}\right) \geq 0$ and $\alpha \leq \alpha(\beta)$. 
Next, we prove that $\mathrm{Q}_{\alpha, \beta}(x)>1 / 2$ for all $x \in(0, \pi / 2)$ if $-1 \leq \beta<0$ and $\alpha \leq \alpha(\beta)$. It follows from $-1 \leq \beta<0$ and $\alpha \leq \alpha(\beta)$ together with Lemma 2.4(3) that

$$
\alpha \leq \alpha(-1)=\alpha_{0}, \quad \beta \leq \beta(\alpha) .
$$

Therefore, $\mathrm{Q}_{\alpha, \beta}(x)>1 / 2$ for all $x \in(0, \pi / 2)$ follows from Lemma 2.6(4) and (2.28).

(3) If $\beta \leq-37 / 35$ and $\mathrm{Q}_{\alpha, \beta}(x)>1 / 2$ for all $x \in(0, \pi / 2)$, then from (2.5)-(2.7) we have

$$
\lim _{x \rightarrow 0^{+}} x^{-2}\left[\mathrm{Q}_{\alpha, \beta}(x)-\frac{1}{2}\right]=\lim _{x \rightarrow 0^{+}} x^{-2}\left[-\frac{5 \alpha+10 \beta+12}{120} x^{2}+o\left(x^{2}\right)\right]=-\frac{5 \alpha+10 \beta+12}{120} \geq 0
$$

which implies that $\alpha \leq-2 \beta-12 / 5$.

If $\beta \leq-37 / 35$ and $\alpha \leq-2 \beta-12 / 5$, then $\mathrm{Q}_{\alpha, \beta}(x)>1 / 2$ for all $x \in(0, \pi / 2)$ follows from (2.8) and Lemma 2.3(3).

(4) If $\beta \leq-37 / 35$ and $\mathrm{Q}_{\alpha, \beta}(x)<1 / 2$ for all $x \in(0, \pi / 2)$, then (2.11) and Lemma 2.4(2) lead to the conclusion that $\mathrm{D}\left(\alpha, \beta ; \frac{\pi}{2}^{-}\right) \leq 0$ and $\alpha \geq \alpha(\beta)$.

Next, we prove that $\mathrm{Q}_{\alpha, \beta}(x)<1 / 2$ for all $x \in(0, \pi / 2)$ if $\beta \leq-37 / 35$ and $\alpha \geq \alpha(\beta)$. It follows from $\beta \leq-37 / 35$ and $\alpha \geq \alpha(\beta)$ together with Lemma 2.4(3) that

$$
\alpha \geq \alpha\left(-\frac{37}{35}\right)=\alpha_{0}^{*}, \quad \beta \geq \beta(\alpha)
$$

Therefore, the desired result follows from Lemma 2.6(2) and (2.29).

\section{Main results}

Let $\alpha, \beta \in \mathbb{R}$ with $\alpha \beta(\alpha+2 \beta) \neq 0$ and $\mathrm{Q}_{\alpha, \beta}(x)$ be defined by (2.7), then we clearly see that the generalized Wilker-type inequality

$$
\frac{2 \beta}{\alpha+2 \beta}\left(\frac{\sin x}{x}\right)^{\alpha}+\frac{\alpha}{\alpha+2 \beta}\left(\frac{\tan x}{x}\right)^{\beta}-1>0
$$

holds for all $x \in(0, \pi / 2)$ if and only if $\mathrm{Q}_{\alpha, \beta}(x)<1 / 2$ and $\alpha \beta(\alpha+2 \beta)>0$ or $\mathrm{Q}_{\alpha, \beta}(x)>1 / 2$ and $\alpha \beta(\alpha+2 \beta)<0$, while the generalized Wilker-type inequality

$$
\frac{2 \beta}{\alpha+2 \beta}\left(\frac{\sin x}{x}\right)^{\alpha}+\frac{\alpha}{\alpha+2 \beta}\left(\frac{\tan x}{x}\right)^{\beta}-1<0
$$

holds for all $x \in(0, \pi / 2)$ if and only if $\mathrm{Q}_{\alpha, \beta}(x)<1 / 2$ and $\alpha \beta(\alpha+2 \beta)<0$ or $\mathrm{Q}_{\alpha, \beta}(x)>1 / 2$ and $\alpha \beta(\alpha+2 \beta)>0$.

From Lemmas 2.6 and 2.7 together with inequalities (3.1) and (3.2) we get Theorems 3.1 and 3.2 immediately.

Theorem 3.1 Let $\alpha, \beta \in \mathbb{R}$ with $\alpha \beta(\alpha+2 \beta) \neq 0, \beta(\alpha), \alpha_{0}$ and $\alpha_{0}^{*}$ be defined by (2.12) and (2.13), respectively. Then the following statements are true:

(1) If $\alpha \geq-2 / 7$, then inequality (3.1) holds for all $x \in(0, \pi / 2)$ if and only if $(\alpha, \beta) \in\{(\alpha, \beta) \mid \beta \leq-\alpha / 2-6 / 5, \alpha \beta(\alpha+2 \beta)<0\}$ and inequality (3.2) holds for all $x \in(0, \pi / 2)$ if and only if $(\alpha, \beta) \in\{(\alpha, \beta) \mid \beta \leq-\alpha / 2-6 / 5, \alpha \beta(\alpha+2 \beta)>0\}$. 
(2) If $\alpha \geq \alpha_{0}^{*}$, then inequality (3.1) holds for all $x \in(0, \pi / 2)$ if and only if $(\alpha, \beta) \in\{(\alpha, \beta) \mid \beta \geq \beta(\alpha), \alpha \beta(\alpha+2 \beta)>0\}$ and inequality (3.2) holds for all $x \in(0, \pi / 2)$ if and only if $(\alpha, \beta) \in\{(\alpha, \beta) \mid \beta \geq \beta(\alpha), \alpha \beta(\alpha+2 \beta)<0\}$.

(3) If $\alpha \leq-2 / 5$, then inequality (3.1) holds for all $x \in(0, \pi / 2)$ if and only if $(\alpha, \beta) \in\{(\alpha, \beta) \mid \beta \geq-\alpha / 2-6 / 5, \alpha \beta(\alpha+2 \beta)>0\}$ and inequality (3.2) holds for all $x \in(0, \pi / 2)$ if and only if $(\alpha, \beta) \in\{(\alpha, \beta) \mid \beta \geq-\alpha / 2-6 / 5, \alpha \beta(\alpha+2 \beta)<0\}$.

(4) If $\alpha \leq \alpha_{0}$, then inequality (3.1) holds for all $x \in(0, \pi / 2)$ if and only if $(\alpha, \beta) \in\{(\alpha, \beta) \mid \beta \leq \beta(\alpha), \alpha \beta(\alpha+2 \beta)<0\}$ and inequality (3.2) holds for all $x \in(0, \pi / 2)$ if and only if $(\alpha, \beta) \in\{(\alpha, \beta) \mid \beta \leq \beta(\alpha), \alpha \beta(\alpha+2 \beta)>0\}$.

Theorem 3.2 Let $\alpha, \beta \in \mathbb{R}$ with $\alpha \beta(\alpha+2 \beta) \neq 0, \alpha_{0}, \alpha_{0}^{*}$, and $\alpha(\beta)$ be defined by Lemma 2.4 . Then the following statements are true:

(1) If $\beta \geq-1$, then inequality (3.1) holds for all $x \in(0, \pi / 2)$ if and only if $(\alpha, \beta) \in\{(\alpha, \beta) \mid \alpha \geq-2 \beta-12 / 5, \alpha \beta(\alpha+2 \beta)>0\}$ and inequality (3.2) holds for all $x \in(0, \pi / 2)$ if and only if $(\alpha, \beta) \in\{(\alpha, \beta) \mid \alpha \geq-2 \beta-12 / 5, \alpha \beta(\alpha+2 \beta)<0\}$.

(2) If $-1 \leq \beta<0$, then inequality (3.1) holds for all $x \in(0, \pi / 2)$ if and only if $(\alpha, \beta) \in\{(\alpha, \beta) \mid \alpha \leq \alpha(\beta), \alpha \beta(\alpha+2 \beta)<0\}$ and inequality (3.2) holds for all $x \in(0, \pi / 2)$ if and only if $(\alpha, \beta) \in\{(\alpha, \beta) \mid \alpha \leq \alpha(\beta), \alpha \beta(\alpha+2 \beta)>0\}$.

(3) If $\beta \leq-37 / 35$, then inequality (3.1) holds for all $x \in(0, \pi / 2)$ if and only if $(\alpha, \beta) \in\{(\alpha, \beta) \mid \alpha \leq-2 \beta-12 / 5, \alpha \beta(\alpha+2 \beta)<0\} \cup\{(\alpha, \beta) \mid \alpha \geq \alpha(\beta), \alpha \beta(\alpha+2 \beta)>0\}$ and inequality (3.2) holds for all $x \in(0, \pi / 2)$ if and only if $(\alpha, \beta) \in\{(\alpha, \beta) \mid \alpha \leq-2 \beta-12 / 5, \alpha \beta(\alpha+2 \beta)>0\} \cup\{(\alpha, \beta) \mid \alpha \geq \alpha(\beta), \alpha \beta(\alpha+2 \beta)<0\}$.

\section{Competing interests}

The authors declare that they have no competing interests.

\section{Authors' contributions}

All authors contributed equally to the writing of this paper. All authors read and approved the final manuscript.

\section{Author details}

${ }^{1}$ School of Mathematics and Computation Sciences, Hunan City University, Yiyang, 413000, China. ${ }^{2}$ Department of Science and Technology, State Grid Zhejiang Electric Power Research Institute, Hangzhou, 310009, China.

\section{Acknowledgements}

The research was supported by the Natural Science Foundation of China under Grants 11371125, 61374086, and 11401191.

Received: 26 September 2016 Accepted: 2 December 2016 Published online: 13 December 2016

\section{References}

1. Wilker, JB: Problem E3306. Am. Math. Mon. 96(1), 55 (1989)

2. Sumner, JS, Jagers, AA, Vowe, M, Anglesio, J: Inequalities involving trigonometric functions. Am. Math. Mon. 98(3), 264-267 (1991)

3. Wu, S-H, Srivastava, H-M: A weighted and exponential generalization of Wilker's inequality and its applications. Integral Transforms Spec. Funct. 18(7-8), 529-535 (2007)

4. Baricz, Á, Sándor, J: Extensions of the generalized Wilker inequality to Bessel functions. J. Math. Inequal. 2(3), 397-406 (2008)

5. Zhu, L: Some new Wilker-type inequalities for circular and hyperbolic functions. Abstr. Appl. Anal. 2009, Article ID 485842 (2009)

6. Matejíčka, L: Note on two Wilker-type inequalities. Int. J. Open Probl. Comput. Sci. Math. 4(1), 79-85 (2011)

7. Zhu, L: A source of inequalities for circular functions. Comput. Math. Appl. 58(10), 1998-2004 (2009)

8. Yang, Z-H, Chu, Y-M: Sharp Wilker-type inequalities with applications. J. Inequal. Appl. 2014, Article ID 166 (2014)

9. Chu, H-H, Yang, Z-H, Chu, Y-M, Zhang, W: Generalized Wilker-type inequalities with two parameters. J. Inequal. Appl. 2016, Article ID 187 (2016)

10. Yang, Z-H, Chu, Y-M, Zhang, X-H: Sharp Cusa type inequalities with two parameters and their applications. Appl. Math. Comput. 268, 1177-1198 (2015) 